\title{
Analysis of Differential Unitary Space-Time Modulation over Non-Identical MIMO Channels
}

\author{
Meixia Tao \\ Department of Electronic Engineering \\ Shanghai Jiao Tong University \\ Shanghai 200240, P. R. China \\ Email: mxtao@ieee.org
}

\begin{abstract}
We present an analytical study on the error performance of differential unitary space-time modulation (DUSTM) over multiple-input multiple-output (MIMO) channels with nonidentical fading statistics. The channel for each transmit-receive antenna pair is assumed to be independent, non-identically distributed (i.n.i.d), and time-varying Rayleigh fading. We first show that the maximum-likelihood (ML) differential detector of DUSTM over such channels is involved except for differential cyclic group codes. Applying the distribution of quadratic forms of Gaussian vectors, we derive closed-form expressions for the exact error probabilities of two specific unitary classes, namely, cyclic group codes and orthogonal codes. Simple and useful asymptotic bounds are also obtained. Our analysis leads to several general findings.
\end{abstract}

\section{INTRODUCTION}

Space-time coding and modulation has experienced enormous development in the last decade. It is commonly assumed in the literature that the channel in a multiple-input multiple-output (MIMO) system is statistically identical in the spatial domain. This is valid when antennas are co-located and hence different transmit-receive antenna pairs experience identical path loss and shadowing. There are many occasions, however, that the antennas are not necessarily co-located. For instance, in distributed antenna systems [1], [2] the antennas are geographically distributed at different radio ports and are connected together through high-speed cables. Cooperative communications among mobile nodes in a network is another important scenario. After knowing each other's data to be sent, the cooperating nodes form a virtual multiple-antenna system and employ space-time coding in a distributed manner [3], [4]. In these communication settings, each signaling branch can have unequal average channel gains and fade rates, resulting in a distributed MIMO channel model.

The goal of this work is to study the performance of existing space-time codes, in particular differential unitary space-time modulation (DUSTM), when applied in (virtual) MIMO systems with non-identical fading statistics. DUSTM is an efficient non-coherent space-time technique proposed in [5], [6]. It eliminates channel estimation at the receiver, thereby, reducing receiver complexity as well as transmission overhead. A number of unitary space-time signal sets have been designed, including orthogonal codes [7], [8], cyclic group codes [9], and Caley differential codes [10]. Whereas the uniform power allocation in spatial domain for DUSTM is capacity-achieving for traditional independent and identically distributed (i.i.d) fading, it may not be so for non-identically distributed fading. Moreover, the conventional differential detector may no longer be optimal in the maximum-likelihood (ML) sense.

Attempts have been made recently to study the effects of non-identical channels on existing space-time techniques. The bit error probabilities (BEP) of coherent orthogonal spacetime block code (OSTBC) over independent and non-identical distributed (i.n.i.d) Rayleigh/Riciean and Nakagami fading channels are analyzed in [11] and [12], respectively. In [13], the authors derived the BEP of differential OSTBC (i.e. the orthogonal-design based DUSTM) over independent and semiidentically distributed (i.s.i.d) Rayleigh channels, where the non-identical fading occurs at the receiver side only.

In this paper, we extend the previous work in [13] to a general framework of DUSTM over i.n.i.d Rayleigh fading channels. Both maximum-likelihood (ML) and conventional detectors are discussed. We then derive the error performance for two specific unitary classes: cyclic group codes and orthogonal codes. In particular, expressions for the exact pairwise error probabilities (PEP) of differential cyclic group codes with both ML and conventional differential detectors (DD) over time-varying fading channels, and expressions for the exact BEP of differential OSTBC with conventional DD in static fading are derived. Furthermore, simple asymptotic bounds for both codes are obtained. These bounds lead to several useful findings suitable for any DUSTM design.

Notations: $\mathcal{E}[\cdot]$ denotes expectation over the random variables within the brackets; $\operatorname{Re} \operatorname{Tr}(\mathbf{A})$ stands for the real part of the trace of matrix A; Superscripts $(\cdot)^{T},(\cdot)^{*}$, and $(\cdot)^{H}$ denote transpose, conjugate, and conjugate transpose, respectively. Notation $\odot$ represents the Hadamard product. Res $[f(x), p]$ denotes the residue of function $f(x)$ at pole $x=p$.

\section{SySTEM MODEL}

Consider a communication system with $M$ transmit and $N$ receive antennas over a flat Rayleigh fading channel. The antenna elements at both the transmitter side and receiver side are not necessarily co-located. At each time block $k$, a set of $\log _{2} L$ information bits are mapped onto a data matrix $\mathbf{D}[k] \in \mathcal{V}$, where $\mathcal{V}=\left\{\mathbf{D}_{i}, 0 \leq i<L\right\}$ denotes a unitary space-time signal constellation with cardinality $L$. 
Each element of $\mathcal{V}$ is an $M \times M$ unitary matrix, satisfying $\mathbf{D}_{i} \mathbf{D}_{i}^{H}=\mathbf{I}_{M}$, for $0 \leq i<L$. For the special case of differential cyclic group codes [6], [9], the constellation set $\mathcal{V}$ forms a group under matrix multiplication and each element of it is a diagonal matrix. In the case of differential OSTBC, each element $\mathbf{D}_{i}$ is a linear mapping of a set of $P$ M-ary PSK modulated information symbols and subject to certain orthogonality constraints. Let $\mathbf{S}[k-1]$ denote the $M \times M$ dimensional code matrix at the $(k-1)$-th time block. The data matrix $\mathbf{D}[k]$ is then differentially encoded as

$$
\mathbf{S}[k]=\mathbf{D}[k] \mathbf{S}[k-1],
$$

where the initial code matrix $\mathbf{S}[0]$ is an arbitrary unitary matrix. The actual signal matrix to be transmitted at time block $k$ over $M$ antennas is given by

$$
\mathbf{X}[k]=\sqrt{E_{s}} \mathbf{S}[k] \mathbf{\Sigma}^{1 / 2},
$$

where $E_{s}$ is the total transmit power, and $\boldsymbol{\Sigma}^{1 / 2}=$ $\operatorname{diag}\left\{\sqrt{\varepsilon_{1}}, \ldots, \sqrt{\varepsilon_{M}}\right\}$ is the power allocation matrix. The power allocation coefficients $\varepsilon_{m}$ 's are subject to the constraint $\sum_{m=1}^{M} \varepsilon_{m}=M$, whose optimization is investigated in [14].

Since the transmission is on a per block basis, we assume the channel is block-wise time-varying with each block containing $M$ symbol intervals. Let $\mathbf{H}[k]$ denote the $M \times N$ channel gain matrix of the $k$-th transmission block, where the $(m, n)$-th entry $h_{m n}[k]$ represents the fading coefficient from the $m$-th transmit antenna to the $n$-th receive antenna. Each $\left\{h_{m n}[k]\right\}_{k}$ is modeled as a complex Gaussian wide-sense stationary random process with zero mean and autocorrelation function $2 R_{m n}[l]=\mathcal{E}\left[h_{m n}[k] h_{m n}^{*}[k-l]\right]$, and is independent for different $m$ and $n$. The channel variance and block correlation coefficient are denoted as $\sigma_{m n}^{2}=2 R_{m n}[0]$ and $\rho_{m n}=R_{m n}[1] / R_{m n}[0]$, respectively.

Let $\mathbf{Y}[k]$ denote the $M \times N$ received signal matrix from $N$ antennas at the $k$-th transmission block. It is modeled as

$$
\mathbf{Y}[k]=\mathbf{X}[k] \mathbf{H}[k]+\mathbf{W}[k],
$$

where $\mathbf{W}[k]$ is the additive white Gaussian noise matrix whose entries are i.i.d with zero mean and variance $N_{0}$.

\section{DifFEREnTial Detection}

In this section we first discuss a general structure of the ML differential detector for DUSTM over the non-identical MIMO channel considered in this paper. Two simplified detectors under certain constraints are then discussed. For notation brevity, we define $\gamma_{0}=E_{s} / N_{0}$ as the total transmit SNR, and define $\gamma_{m n}=\varepsilon_{m} \sigma_{m n}^{2} \gamma_{0}$ as the SNR on the branch between transmit antenna $m$ and receive antenna $n$. In our high SNR assumption, all $\gamma_{m n}$ 's approach infinity as $\gamma_{0} \rightarrow \infty$, but the ratios between one other are kept constant and finite. We also omit the time index $k$ in the data matrix $\mathbf{D}[k]$ and rewrite $\mathbf{S}[k-1]$ as $\mathbf{S}_{-1}$ hereafter as only one data matrix is processed at one time.

\section{A. ML Detection for a General Constellation}

Define $\mathbf{Y}=\left[\mathbf{Y}^{T}[k-1], \mathbf{Y}^{T}[k]\right]^{T}$. Given $\mathbf{D}$ and $\mathbf{S}_{-1}$, it can be shown easily that the column vectors of $\mathbf{Y}$, denoted as $\mathbf{y}_{n}$ for $n=1, \ldots, N$, are mutually independent Gaussian vectors with zero mean and covariance

$$
\begin{aligned}
\boldsymbol{\Lambda}_{n}= & \mathcal{E}\left[\mathbf{y}_{n} \mathbf{y}_{n}^{H}\right] \\
= & N_{0} \underbrace{\left[\begin{array}{cc}
\mathbf{S}_{-1} & \mathbf{0} \\
\mathbf{0} & \mathbf{D S} \\
-1
\end{array}\right]}_{\tilde{\mathbf{s}}} \cdot\left[\begin{array}{cc}
\mathbf{K}_{n 0} & \mathbf{K}_{n 1} \\
\mathbf{K}_{n 1} & \mathbf{K}_{n 0}
\end{array}\right] \cdot \underbrace{\left[\begin{array}{cc}
\mathbf{S}_{-1}^{H} & \mathbf{0} \\
\mathbf{0} & \mathbf{S}_{-1}^{H} \mathbf{D}^{H}
\end{array}\right]}_{\tilde{\mathbf{S}}^{H}} \\
& +N_{0} \mathbf{I}_{2 M} .
\end{aligned}
$$

Here, $\mathbf{K}_{n i}$ 's, for $i=0,1$ and $n=1, \ldots, N$, are $M \times M$ diagonal matrices defined as $\mathbf{K}_{n 0}=\operatorname{diag}\left\{\gamma_{1 n}, \ldots, \gamma_{M n}\right\}$ and $\mathbf{K}_{n 1}=\operatorname{diag}\left\{\rho_{1 n} \gamma_{1 n}, \ldots, \rho_{M n} \gamma_{M n}\right\}$. Applying the formula for the determinant of a partitioned matrix, we show that the determinant of the covariance matrix $\boldsymbol{\Lambda}_{n}$ is independent of the data matrix $\mathbf{D}$ and the previous code matrix $\mathbf{S}_{-1}$. The inverse of $\boldsymbol{\Lambda}_{n}$, however, will in general depend not only on $\mathbf{D}$, but also on $\mathbf{S}_{-1}$. Using the Sherman-Morrison-Woodbury formula for the inverse of the matrix of the form $\mathbf{A}+\mathbf{B C D}$ and utilizing the diagonal structure of $\mathbf{K}_{n i}$, we obtain the inverse of $\boldsymbol{\Lambda}_{n}$ as

$$
\left(\boldsymbol{\Lambda}_{n}\right)^{-1}=\frac{1}{N_{0}} \mathbf{I}_{2 M}-\frac{1}{N_{0}} \underbrace{\tilde{\mathbf{S}}}_{\tilde{\mathbf{C}}_{n}} \underbrace{\mathbf{S}^{H},}_{\left.\begin{array}{ll}
\mathbf{C}_{n 0} & \mathbf{C}_{n 1} \\
\mathbf{C}_{n 1} & \mathbf{C}_{n 0}
\end{array}\right]}
$$

where matrices $\mathbf{C}_{n i}$, for $i=0,1$ and $n=1, \ldots, N$, are also diagonal, whose $m$-th diagonals, for $m=1, \ldots, M$, are

$$
\begin{aligned}
{\left[\mathbf{C}_{n 0}\right]_{m} } & =\frac{\gamma_{m n}\left[1+\gamma_{m n}\left(1-\rho_{m n}^{2}\right)\right]}{\left(1+\gamma_{m n}\right)^{2}-\left(\rho_{m n} \gamma_{m n}\right)^{2}}, \\
{\left[\mathbf{C}_{n 1}\right]_{m} } & =\frac{\gamma_{m n} \rho_{m n}}{\left(1+\gamma_{m n}\right)^{2}-\left(\rho_{m n} \gamma_{m n}\right)^{2}} .
\end{aligned}
$$

The ML differential detector of $\mathbf{D}$ is to choose the candidate $\hat{\mathbf{D}} \in \mathcal{V}$ that maximizes the joint likelihood function of the received signal matrix $\mathbf{Y}$ over all possible $\mathbf{S}_{-1}$. Applying (4), we arrive at the quadratic-form based ML differential detector for a general unitary constellation

$$
\hat{\mathbf{D}}_{\mathrm{ML}}=\arg \max _{\mathbf{D} \in \mathcal{V}} \max _{\mathbf{S}_{-1}} \sum_{n=1}^{N} \mathbf{y}_{n}^{H} \tilde{\mathbf{S}} \tilde{\mathbf{C}}_{n} \tilde{\mathbf{S}}^{H} \mathbf{y}_{n} .
$$

\section{B. ML Detection for Cyclic Group Codes}

Because of the diagonal structure inherent in cyclic groups, the code matrix $\mathbf{S}_{-1}$ is always diagonal as long as the initial matrix $\mathbf{S}[0]$ is diagonal, say $\mathbf{I}_{M}$. Since multiplication commutes for diagonal matrices, we have $\mathbf{S}_{-1} \mathbf{C}_{n i} \mathbf{S}_{-1}^{H}=\mathbf{C}_{n i}$, for all $i$ and $n$. Therefore, the ML detector for cyclic group codes reduces to

$$
\hat{\mathbf{D}}_{\mathrm{ML}, \mathrm{c}}=\arg \max _{\mathbf{D} \in \mathcal{V}} \sum_{n=1}^{N} \mathbf{y}_{n}^{H}\left[\begin{array}{c}
\mathbf{I} \\
\mathbf{C}_{n 1} \mathbf{D}
\end{array}\right]\left[\mathbf{I} \mathbf{C}_{n 1} \mathbf{D}^{H}\right] \mathbf{y}_{n}
$$

which can be further expressed as:

$$
\hat{\mathbf{D}}_{\mathrm{ML}, \mathrm{c}}=\arg \max _{\mathbf{D} \in \mathcal{V}} \operatorname{Re} \operatorname{Tr}\left\{(\mathbf{Y}[k-1] \odot \mathbf{W})^{H} \mathbf{D}^{H} \mathbf{Y}[k]\right\}
$$


where $\mathbf{W}$ is an $M \times N$ matrix with the $(m, n)$-th entry formed by the $m$-th diagonal of $\mathbf{C}_{n 1}$ and repeated as:

$$
w_{m n}=\frac{\gamma_{m n} \rho_{m n}}{\left(1+\gamma_{m n}\right)^{2}-\left(\rho_{m n} \gamma_{m n}\right)^{2}} .
$$

It is clear from (7) that the ML detector for cyclic group codes resembles the conventional DD but applies a weight $w_{m n}$ to the $(m, n)$-th element of $\mathbf{Y}[k-1]$. The resulting Hadamard product $\mathbf{Y}[k-1] \odot \mathbf{W}$ behaves as the equivalent channel matrix as in coherent receivers. .

\section{Asymptotically Optimal Detection for a General Constel- lation in Static Channels}

In static fading channels the channel coefficients are assumed to remain unchanged over the duration of two transmission blocks. Therefore it has $\rho_{m n}=1$ for all $m$ and $n$. In the limit $\gamma_{m n} \rightarrow \infty$ for all $m$ and $n$, the matrices $\mathbf{C}_{n 0}$ and $\mathbf{C}_{n 1}$ all approach (1/2) $\mathbf{I}_{M}$. Applying these into (5), we obtain the asymptotically optimal detector:

$$
\hat{\mathbf{D}}_{\mathrm{AO}}=\arg \max _{\mathbf{D} \in \mathcal{V}} \sum_{n=1}^{N} \mathbf{y}_{n}^{H}\left[\begin{array}{l}
\mathbf{I} \\
\mathbf{D}
\end{array}\right]\left[\begin{array}{l}
\mathbf{I} \\
\mathbf{D}^{H}
\end{array}\right] \mathbf{y}_{n} .
$$

This is identical to the conventional DD [6]. Hence we conclude that the conventional DD is suboptimal in i.n.i.d time-varying channels but asymptotically optimal at high SNR if the channel varies slowly enough.

\section{ERROR PROBABILITY ANALYSIS}

In this section, we derive the error performance of DUSTM with two specific constellation designs: cyclic group codes and orthogonal codes. Through the analysis, we obtain several general findings that are applicable to any DUSTM design.

\section{A. Pairwise Error Probability for Cyclic Group Codes}

Let us assume that the transmitted data matrix is $\mathbf{D}_{i}$ and the erroneously decoded matrix is $\mathbf{D}_{j}$. Based on the quadratic form of the ML and suboptimal detectors given in (6) and (9) respectively, the PEP can be expressed as

$$
P_{e, i j}=P\left(z_{i j}<0 \mid \mathbf{D}_{i}\right),
$$

where the pairwise decision variable $z_{i j}$ is defined as $z_{i j}=$ $\sum_{n=1}^{N} \mathbf{y}_{n}^{H} \boldsymbol{\Omega}_{i j, n} \mathbf{y}_{n}$ with the Hermitian matrix $\boldsymbol{\Omega}_{i j, n}$ given by

$$
\boldsymbol{\Omega}_{i j, n}=\left[\begin{array}{cc}
\mathbf{0} & \mathbf{C}_{n 1}\left(\mathbf{D}_{i}-\mathbf{D}_{j}\right)^{H} \\
\mathbf{C}_{n 1}\left(\mathbf{D}_{i}-\mathbf{D}_{j}\right) & \mathbf{0}
\end{array}\right]
$$

for ML detection, or

$$
\boldsymbol{\Omega}_{i j, n}=\boldsymbol{\Omega}_{i j}=\left[\begin{array}{cc}
\mathbf{0} & \left(\mathbf{D}_{i}-\mathbf{D}_{j}\right)^{H} \\
\left(\mathbf{D}_{i}-\mathbf{D}_{j}\right) & \mathbf{0}
\end{array}\right]
$$

for conventional detection. Since each vector $\mathbf{y}_{n}$ is independent and zero-mean complex Gaussian distributed, the pairwise decision variable $z_{i j}$ is a quadratic form of Gaussian vectors. Therefore, the evaluation of PEP can be carried out by using the well-established techniques in, e.g., [15, Appendix A]. We summarize the results in the following proposition, the proof of which is sketched in the Appendix.
Proposition 1: The exact pairwise error probability $P_{e, i j}$ of differential cyclic group codes over i.n.i.d time-varying Rayleigh fading channels is

$$
\begin{gathered}
P_{e, i j}=-\sum_{\substack{1 \leq k \leq M \\
1 \leq l \leq N}} \operatorname{Res}\left\{\frac{1}{s \prod_{m} \prod_{n} a_{m n}\left[\frac{1}{4}+\frac{1}{a_{m n}}-\left(\frac{s}{b_{m n}}-\frac{1}{2}\right)^{2}\right]},\right. \\
s=b_{k l}\left[\frac{1}{2}+\sqrt{\left.\frac{1}{4}+\frac{1}{a_{k l}}\right]}\right\},
\end{gathered}
$$

where

$$
a_{m n}=\frac{\left(\rho_{m n} \gamma_{m n}\right)^{2}}{\left(1+\gamma_{m n}\right)^{2}-\left(\rho_{m n} \gamma_{m n}\right)^{2}} d_{i j, m}
$$

with $d_{i j, m}$ being the $m$-th diagonal entry of the difference $\operatorname{matrix}\left(\mathbf{D}_{i}-\mathbf{D}_{j}\right)\left(\mathbf{D}_{i}-\mathbf{D}_{j}\right)^{H}$, and

$$
b_{m n}=\left\{\begin{array}{ll}
1, & \text { ML detector } \\
w_{m n} \text { in }(8), & \text { conv. detector }
\end{array} .\right.
$$

In the case of ML differential detection, an alternative expression is given by

$$
P_{e, i j}=\frac{1}{\pi} \int_{0}^{\pi / 2} \prod_{n=1}^{N} \prod_{m=1}^{M}\left(1+\frac{a_{m n}}{4 \sin ^{2} \theta}\right)^{-1} d \theta .
$$

In the above proposition, assuming static fading channels with $\rho_{m n}=1$ and taking the limit $\gamma_{0} \rightarrow \infty$, one finds from (14) and (8) that $a_{m n} \rightarrow \gamma_{m n} d_{i j, m} / 2$ and $w_{m n} \rightarrow 1 / 2$. As a result, the $M N$ poles, where the residues are evaluated, all approach the constant 1 for ML DD or the constant $1 / 2$ for conventional DD. After applying the definition of residue in (13), we arrive at the asymptotic results as follows for both ML and conventional DD.

Corollary 1: The asymptotic PEPs of differential cyclic group codes at high SNR with ML and conventional differential detection over i.n.i.d static Rayleigh fading channels are the same and given by

$$
\lim _{\gamma_{0} \rightarrow \infty} P_{e, i j}=\left(\begin{array}{c}
2 M N-1 \\
M N
\end{array}\right)\left(\prod_{m=1}^{M} d_{i j, m}\right)^{-N}\left(\frac{\gamma_{\mathrm{gm}}}{2}\right)^{-M N}
$$

where $\gamma_{\mathrm{gm}}$ is the geometric mean of $\left\{\gamma_{m n}\right\}$, given by $\gamma_{\mathrm{gm}}=$ $\left(\prod_{n=1}^{N} \prod_{m=1}^{M} \gamma_{m n}\right)^{1 / M N}$, and $\left(\begin{array}{c}2 M N-1 \\ M N\end{array}\right)$ denotes the binomial coefficient.

Corollary 1 leads to several insights. First, the result that the asymptotic PEPs of ML and conventional DD are the same is consistent with the finding in Section III-C that the conventional DD is in fact asymptotically optimal for a general constellation without assuming a specific signal structure. Using the arithmetic-geometric-mean inequality, we can see that the non-identical channel distribution will degrade the error performance compared with the identical case if the total received SNR is kept the same. Furthermore, after rewriting $\gamma_{\mathrm{gm}}$ as $\gamma_{0}\left(\prod_{m=1}^{M} \varepsilon_{m}\right)^{1 / M}\left(\prod_{n=1}^{N} \prod_{m=1}^{M} \sigma_{m n}^{2}\right)^{1 / M N}$, where $\varepsilon_{m}$ is the power allocation coefficient defined in (1) subject to the constraint $\sum_{m=1}^{M} \varepsilon_{m}=M$, it follows that $\gamma_{\mathrm{gm}}$ is maximized when $\varepsilon_{m}=1$ for all $m$. Therefore, equal power allocation is asymptotically optimal in static channels. 


\section{B. Bit Error Probability for Orthogonal Codes}

In this subsection we derive the error performance of differential OSTBC. For analytical tractability, only the conventional DD and static channels are considered. Since the data matrix D is a linear combination of $P$ information symbols as mentioned in Section II, the differential detector (9) reduces to $P$ independent symbol-by-symbol detectors. The details are given in [8] or [13, eq.(12)]. Hence, instead of PEP, BEP is derived.

As shown in [13, eq.(15)-(17)], the BEP conditioned on symbol $s_{p}$ is the same for all $p$, and can be expressed as $P_{b}(\alpha)=P\left(z_{p}(\alpha)<0 \mid s_{p}=1\right)$. Here the decision phasor $z_{p}(\alpha)$, for $p=1, \ldots, P$, is defined as $z_{p}(\alpha)=$ $\sum_{n=1}^{N} \mathbf{y}_{n}^{H} \boldsymbol{\Omega}_{p} \mathbf{y}_{n}$, with the Hermitian matrix $\boldsymbol{\Omega}_{p}$ given by [13, eq.(18)] and $\alpha$ is some angle that depends on the symbol modulation scheme. For BPSK, the exact BEP is obtained by letting $\alpha=0$, and for QPSK with Gray mapping we have $\alpha=-\pi / 4$. Through deriving the eigenvalues of the matrix $\Lambda_{n} \boldsymbol{\Omega}_{p}$, we can obtain the distribution of $z_{p}(\alpha)$ and hence the expression of $P_{b}(\alpha)$. The detailed proof is skipped.

Proposition 2: The exact bit error probability $P_{b}(\alpha)$ of differential OSTBC over i.n.i.d static Rayleigh fading channels with conventional differential detection is

$$
\begin{gathered}
P_{b}(\alpha)=-\sum_{\substack{1 \leq k \leq M \\
1 \leq l \leq N}} \operatorname{Res}\left\{\frac{1}{s \prod_{n} \prod_{m}\left[1+2 c \gamma_{m n} s-\left(1+2 \gamma_{m n}\right) s^{2}\right]}\right. \\
\left.s=\frac{c \gamma_{k l}}{1+2 \gamma_{k l}}\left[1+\sqrt{1+\frac{1+2 \gamma_{k l}}{c^{2} \gamma_{k l}^{2}}}\right]\right\}
\end{gathered}
$$

where $c=\cos \alpha / \sqrt{P}$.

At high SNR, all the $M N$ positive poles approach the constant $c$. Therefore, we obtain the asymptotics of $P_{b}(\alpha)$ as follows by applying the definition of residue.

Corollary 2: The asymptotic BEP of differential OSTBC over i.n.i.d static Rayleigh fading channels with conventional differential detection is

$$
\lim _{\gamma_{0} \rightarrow \infty} P_{b}(\alpha)=\left(\begin{array}{c}
2 M N-1 \\
M N
\end{array}\right)\left(\frac{2 \cos ^{2} \alpha}{P} \gamma_{\mathrm{gm}}\right)^{-M N}
$$

where $\gamma_{\mathrm{gm}}$ is the geometric mean of $\left\{\gamma_{m n}\right\}$.

The implications in Corollary 2 are the same as those in Corollary 1 . Therefore, we readily extend these remarks to the general DUSTM: (1) Non-identical fading degrades the error performance compared with the identical case given the same total received SNR; (2) Equal power allocation is asymptotically optimal in i.n.i.d static fading channels.

\section{Numerical Results}

In this section we present some numerical examples to confirm our analytical findings in previous sections. A system with $M=2$ transmit antennas and $N=2$ receive antennas is considered. The unequal average channel gains are generated using the Kronecker model [13]. In specific, the $M N \times M N$ diagonal matrix $\boldsymbol{\Delta}$ with $\sigma_{m n}^{2}$ on the $[(n-1) M+m]$-th diagonal is decomposed as $\boldsymbol{\Delta}=\boldsymbol{\Delta}_{T} \otimes \boldsymbol{\Delta}_{R}$, where $\boldsymbol{\Delta}_{T}$ and $\boldsymbol{\Delta}_{R}$ are, respectively, the $M \times M$ and $N \times N$ diagonal matrices inducing non-identical fading parameters at the transmitter and receiver. The sum of the average channel gains is normalized so that $\operatorname{Tr}\left\{\boldsymbol{\Delta}_{T}\right\}=M$ and $\operatorname{Tr}\left\{\boldsymbol{\Delta}_{R}\right\}=N$. In our example, we specify $\boldsymbol{\Delta}_{T}=\boldsymbol{\Delta}_{R}=\operatorname{diag}\left(\frac{1}{5}, \frac{9}{5}\right)$. The set of average channel gains is thus given by $\left\{\sigma_{11}^{2}=1 / 25, \sigma_{12}^{2}=\sigma_{21}^{2}=9 / 25, \sigma_{22}^{2}=81 / 25\right\}$. A block-wise time-varying channel is generated in simulation based on Jakes model with normalized Doppler frequency $f_{d} T_{s}$. The same $f_{d} T_{s}$ is assumed for all transmit-receive antenna pairs for simplicity. The block correlation coefficient used for analytical study is taken as $\rho=\mathcal{J}_{0}\left(2 \pi f_{d} T_{s} M\right)$, where $\mathcal{J}_{0}(\cdot)$ is the zeroth order Bessel function of the first kind.

Fig. 1 shows the analytical PEP of the cyclic group code $\mathcal{V}_{2,4}$ at rate $1-\mathrm{bit} / \mathrm{s} / \mathrm{Hz}$ [6]. It is seen that with ML detector, the pairwise error floor in the time-varying channel $\left(f_{d} T_{s}=0.02\right.$ or $\rho=0.98427)$ is significantly reduced and approaches the floor in i.i.d channels. The pairwise error probabilities achieved by ML and conventional detectors are almost the same at low SNR in time-varying fading and at all SNR in static fading. Fig. 2 illustrates the simulated overall BEP of this cyclic group code in the time-varying channel. The BEP union bound obtained analytically based on the exact PEP is also given. The closeness between the analytical union bound and actual BEP validates our theoretical analysis in Proposition 1.

In Fig. 3, we present the BEP results of differential OSTBC with QPSK modulation using conventional DD in static fading. The analytical results for i.n.i.d channel are validated by simulations. By comparing with the results over i.s.i.d channel (18) with $\sigma_{11}^{2}=\sigma_{21}^{2}=1 / 5$ and $\sigma_{12}^{2}=\sigma_{22}^{2}=9 / 5$, and i.i.d channel with $\sigma_{m n}^{2}=1$ for all $m, n$, it is seen that the i.n.i.d channel yields the worst performance, as expected.

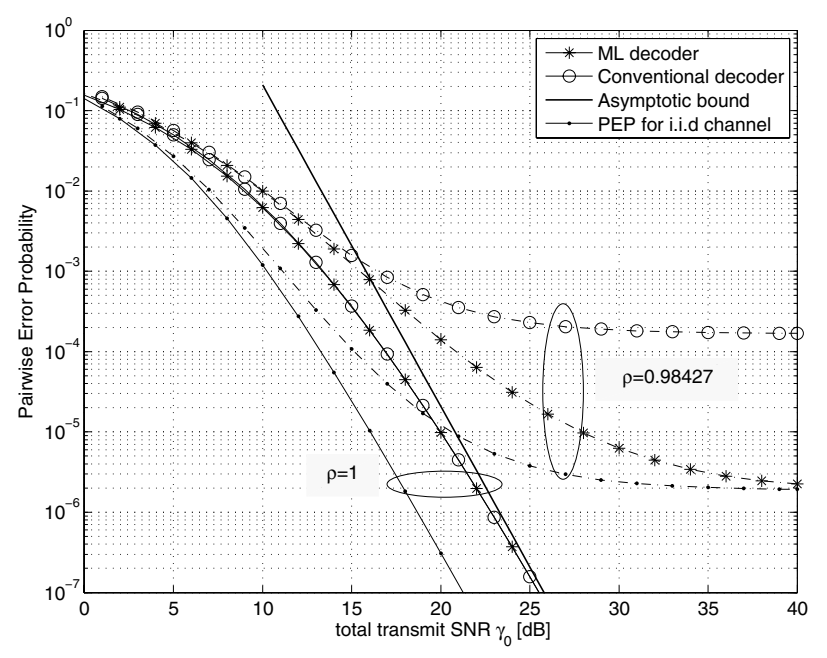

Fig. 1. Analytical PEP between $\mathbf{D}_{0}$ and $\mathbf{D}_{1}$ of differential cyclic group code $\mathcal{V}_{2,4}$ over i.n.i.d Rayleigh fading.

\section{CONCLUSION}

The effects of non-identical fading statistics in MIMO channels on the performance of DUSTM were investigated. We found that the ML differential detection generally requires joint optimization of the current data matrix and the previously transmitted signal matrix. However for DUSTM with cyclic 


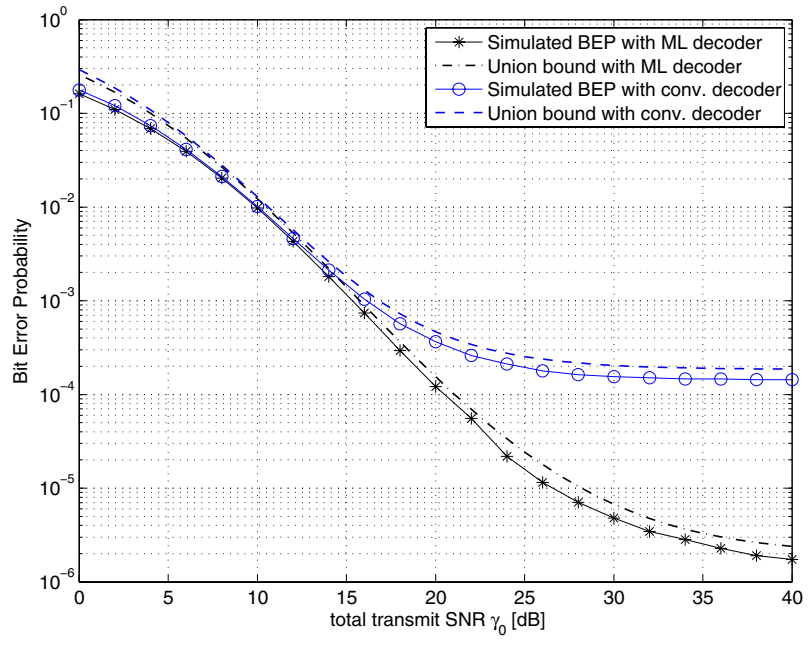

Fig. 2. Simulated BEP and its analytical union bound of differential cyclic group code $\mathcal{V}_{2,4}$ over i.n.i.d Rayleigh fading with $f_{d} T_{s}=0.02$.

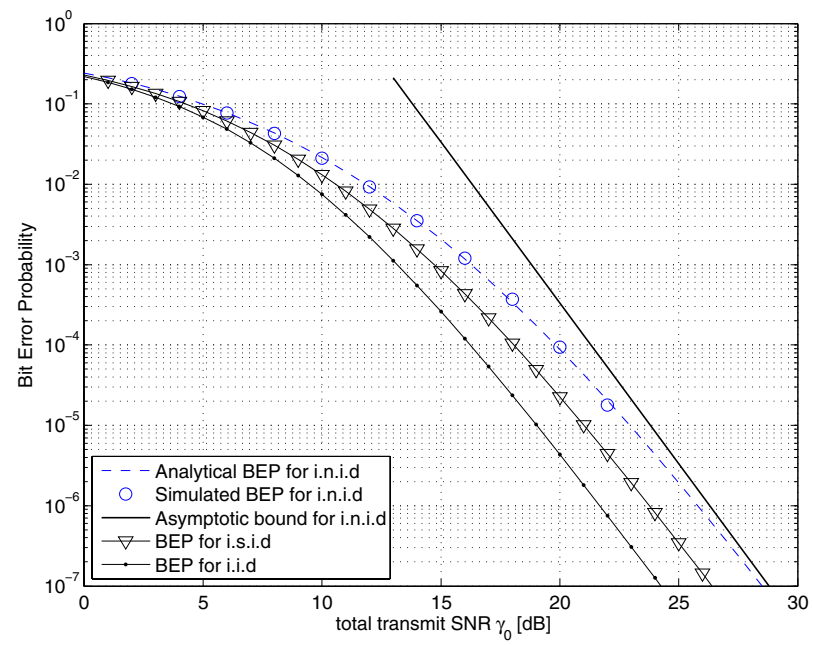

Fig. 3. BEP performance of differential OSTBC with QPSK modulation over different channels with $\rho=1$.

group design, the ML detector is much simplified and is similar to the conventional one but applies fading statisticsdependent weights. Through the analysis of error probability for cyclic group codes and orthogonal codes, we obtained several insights about the effects of non-identical fading on general DUSTM schemes. In particular, we showed that nonidentical channel gain distribution degrades error performance compared with the identical case. We also concluded that equal power allocation is asymptotically optimal in static fading.

\section{APPENDIX I}

\section{PROOF OF PROPOSITION 1}

By applying the result in [16], the characteristic function (CF) of $z_{i j}$ in the quadratic form of Gaussian vectors can be written as

$$
\phi_{z_{i j}}(s)=\mathcal{E}\left[e^{-s z_{i j}}\right]=\frac{1}{\prod_{n=1}^{N} \operatorname{det}\left(\mathbf{I}+s \boldsymbol{\Lambda}_{n} \boldsymbol{\Omega}_{i j, n}\right)} .
$$

Substituting (3) and (11) (or (12)) into (20), we obtain

$$
\phi_{z_{i j}}(s)=\left[\prod_{n=1}^{N} \prod_{m=1}^{M} a_{m n}\left(\frac{1}{4}+\frac{1}{a_{m n}}-\left(\frac{s}{b_{m n}}-\frac{1}{2}\right)^{2}\right)\right]^{-1}
$$

where $a_{m n}$ and $b_{m n}$ are given in (14) and (15), respectively. After inverting the Laplace transform, we express $P_{e, i j}$ defined in (10) as $[15$, Appendix A]

$$
P_{e, i j}=\frac{1}{2 \pi j} \int_{-j \infty+\eta}^{j \infty+\eta} \frac{\phi_{z_{i j}}(s)}{s} d s,
$$

where $\eta>0$ is within the region of convergence. This integral can be solved using Cauchy's theorem in terms of residues:

$$
P_{e, i j}=-\sum_{p_{i}>0} \operatorname{Res}\left[\frac{\phi_{z_{i j}}(s)}{s}, s=p_{i}\right],
$$

where $p_{i}$ 's are all the positive poles of $\phi_{z_{i j}}(s)$. Finally, substituting (21) into (23) yields $P_{e, i j}$ expressed more explicitly in (13).

In the case of ML differential detection with $b_{m n}=1$, we can choose $\eta=1 / 2$ for the integration contour in (22). Then, with a change of variables, we obtain

$$
P_{e, i j}=\frac{1}{2 \pi j} \int_{-j \infty}^{j \infty} \frac{d s}{\left(s+\frac{1}{2}\right) \prod_{m=1}^{M} \prod_{n=1}^{N} a_{m n}\left(\frac{1}{4}+\frac{1}{a_{m n}}-s^{2}\right)} .
$$

Now we let $s=j w$ and the integration becomes along the real axis. By further letting $w=\tan \theta / 2$, an alternative expression of $P_{e, i j}$ in the form of finite integral is obtained in (16).

\section{REFERENCES}

[1] W. Roh and A. Paulraj, "Outage performance of the distributed antenna systems in a composite fading channels," in Proc. IEEE VTC'02 Fall, 2002.

[2] H. Zhang and H. Dai, "On the capacity of distributed MIMO systems," in Proc. CISS'04, 2004.

[3] J. N. Laneman and G. W. Wornell, "Distributed space-time coded protocols for exploiting cooperative diversity in wireless networks," IEEE Trans. Info. Theory, vol. 49, no. 10, pp. 2415-2425, Oct. 2003.

[4] S. Yiu, R. Schober, and L. Lampe, "Distributed space-time block coding," IEEE Trans. Comm., vol. 54, no. 7, pp. 1195-1206, July 2006.

[5] B. L. Hughes, "Differential space-time modulation," IEEE Trans. on Info. Theory, vol. 46, no. 7, pp. 2567-2578, Nov. 2000.

[6] B. M. Hochwald and W. Sweldens, "Differential unitary space-time modulation," IEEE Trans. on Comm., vol. 48, no. 12, pp. 2041-2052, Dec. 2000.

[7] V. Tarokh and H. Jafarkhani, "A differential detection scheme for transmit diversity," IEEE Journal on Selected Areas in Comm., vol. 18, no. 7, pp. 1169-1174, 2000.

[8] G. Ganesan and P. Stoica, "Differential detection based on space-time block codes," Wireless Personal Comm., vol. 21, pp. 163-180, 2002.

[9] B. L. Hughes, "Optimal space-time constellations from groups," IEEE Trans. Info. Theory, vol. 49, no. 2, pp. 401-410, Feb. 2003.

[10] B. Hassibi and B. M. Hochwald, "Cayley differential unitary space-time codes," IEEE Trans. Info. Theory, vol. 48, no. 6, pp. 1485-1503, June 2002.

[11] J. He and P. Y. Kam, "On the performance of orthogonal spacetime block codes over independent, nonidentical Rayleigh/Ricean fading channels," in Proc. IEEE GLOBECOM'06, 2006.

[12] H. Zhao, Y. Gong, Y. L. Guan, C. L. Law, and Y. Tang, "Spacetime block codes in Nakagami fading channels with non-identical $m$ distributions," in Proc. IEEE WCNC'07, Hong Kong, March 2007.

[13] M. Tao and P. Y. Kam, "Analysis of differential orthogonal space-time block codes over semi-identical MIMO fading channels," IEEE Trans. on Comm., vol. 55, no. 2, pp. 282-291, Feb. 2007.

[14] M. Tao, "Effects of non-identical rayleigh fading on differential unitary space-time modulation," submitted to IEEE Trans. on Comm.

[15] M. Brehler and M. K. Varanasi, "Asymptotic error probability analysis of quadratic receivers in Rayleigh-fading channels with applications to a unified analysis of coherent and noncoherent space-time receivers," IEEE Trans. on. Info. Theory, vol. 47, no. 6, pp. 2383-2399, Sept 2001.

[16] G. L. Turin, "The characteristic function of hermitian quadratic forms in complex normal variables," Biometrika, vol. 47, pp. 199-201, 1960. 